\title{
SGLT2 inhibitor dapagliflozin limits podocyte damage in proteinuric nondiabetic nephropathy
}

Paola Cassis, ${ }^{1}$ Monica Locatelli, ${ }^{1}$ Domenico Cerullo, ${ }^{1}$ Daniela Corna, ${ }^{1}$ Simona Buelli, ${ }^{1}$ Cristina Zanchi, ${ }^{1}$ Sebastian Villa, ${ }^{1}$ Marina Morigi, ${ }^{1}$ Giuseppe Remuzzi, ${ }^{1,2,3}$ Ariela Benigni, ${ }^{1}$ and Carlamaria Zoja ${ }^{1}$

IIRCCS - Istituto di Ricerche Farmacologiche Mario Negri, Centro Anna Maria Astori, Science and Technology Park Kilometro Rosso, Bergamo, Italy. ${ }^{2}$ Unit of Nephrology and Dialysis, Azienda Socio-Sanitaria Territoriale (ASST) Papa Giovanni XXIII, Bergamo, Italy. ${ }^{3}$ Department of Biomedical and Clinical Sciences, University of Milan, Milan, Italy.

Sodium-glucose cotransporter 2 (SCLT2) inhibitors have pleiotropic properties beyond blood glucose-lowering effects and modify important nonglycemic pathways, leading to end-organ protection. SGLT2 inhibitors display renoprotective effects in diabetic kidney disease, which creates a rationale for testing the therapeutic potential of this drug class in nondiabetic chronic kidney disease. Here, we have shown that dapagliflozin provided glomerular protection in mice with protein-overload proteinuria induced by bovine serum albumin (BSA), to a similar extent as an ACE inhibitor used as standard therapy for comparison. Dapagliflozin limited proteinuria, glomerular lesions, and podocyte dysfunction and loss. We provide the observation that SGLT2 was expressed in podocytes and upregulated after BSA injections. Through in vitro studies with cultured podocytes loaded with albumin we have identified what we believe to be a novel mechanism of action for SCLT2 inhibitor that directly targets podocytes and relies on the maintenance of actin cytoskeleton architecture. Whether SCLT2 inhibitors represent a possible future therapeutic option for some patients with proteinuric glomerular disease who do not have as yet an effective treatment will require ad hoc clinical studies.

Conflict of interest: The authors received funding from AstraZeneca Lab Italia.

Submitted: November 16, 2017

Accepted: June 21, 2018

Published: August 9, 2018

Reference information: JCI Insight. 2018;3(15):e98720. https://doi.org/10.1172/jci. insight. 98720.

\section{Introduction}

Sodium-glucose cotransporter 2 (SGLT2) inhibitors are a class of antihyperglycemic drugs approved for the treatment of type 2 diabetes. These drugs block renal reabsorption of glucose by inhibiting SGLT2, the primary sodium-coupled glucose transporter in the proximal tubules, promoting glycosuria and lowering blood glucose (1-3). Apart from the ability to increase urinary glucose excretion and help control glycemia, SGLT2 inhibitors have additional properties that are potentially relevant for renoprotection in diabetic nephropathy (4).

In the diabetic kidney, glomerular hyperfiltration, which is closely dependent on increased intraglomerular capillary pressure, is a detrimental process that leads to the loss of permselective properties of the glomerular barrier to proteins, resulting in albuminuria and end-stage renal failure $(5,6)$. In diabetic patients, because of a high filtered load of glucose, reabsorption of glucose and sodium is increased in the proximal tubule via SGLT2, resulting in a diminished delivery of sodium to the macula densa (3, $7,8)$. These effects reduce the tubuloglomerular feedback signal and lead to local release of renin and angiotensin II that results in the constriction of the adjacent efferent arteriole, and dilatation of the afferent arteriole, thereby increasing intraglomerular pressure and single-nephron glomerular filtration. Inhibition of SGLT2 increases the delivery of glucose along with sodium to the macula densa, activating the tubuloglomerular feedback and promoting afferent arteriolar constriction, which in turn decreases intraglomerular pressure and hyperfiltration (3, 7-9). Attenuation of glomerular hyperfiltration would translate into the reduction of albuminuria, contributing to reducing the progression of diabetic kidney disease. Clinical studies have shown that SGLT2 inhibitors reduced glomerular hyperfiltration, followed by the stabilization of renal function in the long term, and lowered albuminuria in type 2 diabetes, independently of glycemic control (10-12). Furthermore, in experimental diabetes SGLT2 inhibitors limited albuminuria, mesangial expansion and matrix accumulation, and interstitial fibrosis, via the combined 
Table 1. Systemic and laboratory parameters

\begin{tabular}{|c|c|c|c|c|c|}
\hline Group & Body weight (g) & Diuresis (ml/24 h) & Serum $\mathrm{Na}^{+}(\mathrm{mEq} / \mathrm{l})$ & SBP $(\mathrm{mmHg})$ & Blood glucose (mg/dl) \\
\hline Control & $25.17 \pm 0.37$ & $1.19 \pm 0.13$ & $156.68 \pm 3.57$ & $97.83 \pm 3.36$ & $110.75 \pm 0.85$ \\
\hline BSA + Dapagliflozin & $25.75 \pm 0.59$ & $3.1 \pm 0.26^{\mathrm{B}, \mathrm{C}}$ & $154.27 \pm 1.62$ & $85.88 \pm 3.30^{D}$ & $96.33 \pm 5.44^{\mathrm{C}, \mathrm{E}}$ \\
\hline BSA + Lisinopril & $25.00 \pm 0.38$ & $1.7 \pm 0.30$ & $152.48 \pm 3.28$ & $74.38 \pm 3.99^{\mathrm{B}, \mathrm{E}}$ & $137.25 \pm 8.58^{A}$ \\
\hline
\end{tabular}

Data are the mean \pm SEM. ${ }^{A} P<0.05,{ }^{B} P<0.01$ versus control; ${ }^{C} P<0.01$ versus $B S A+A C E$ inhibitor (lisinopril); ${ }^{D} P<0.05,{ }^{E} P<0.01$ versus $B S A+$ vehicle; ANOVA with Tukey's post hoc test. SBP, systolic blood pressure.

effects on glomerular hemodynamics and inhibition of renal inflammation and oxidative stress (13-16). These findings indicated that SGLT2 inhibitors may modify important nonglycemic pathways, leading to end-organ protection (17). The evidence that the protective effects of SGLT2 inhibitors on the kidney likely occur independently of glucose regulation offers the rationale for testing the therapeutic potential of this drug class in nondiabetic chronic kidney disease (CKD).

In the present study, we investigated the effects of dapagliflozin treatment in an experimental model of proteinuric nondiabetic nephropathy (18). We show that in mice with protein-overload proteinuria, dapagliflozin limited proteinuria and glomerular lesions, in association with the amelioration of podocyte dysfunction and loss. We also demonstrate that mouse podocytes express SGLT2, the levels of which increase with protein overload. In vitro data underlined that podocytes are a target of the SGLT2 inhibitor's protective effect against albumin-induced cytoskeletal remodeling.

\section{Results}

Dapagliflozin's effects on systemic and laboratory parameters in mice with proteinuric nephropathy. Mice with protein-overload proteinuria were treated with vehicle or dapagliflozin and followed up until 23 days after starting bovine serum albumin (BSA) injections. The angiotensin-converting enzyme (ACE) inhibitor lisinopril was used as a standard antiproteinuric therapy (19) for comparison. Systemic and laboratory parameters of the investigated mice are given in Table 1 and Figure 1. No differences in body weight were observed between groups (Table 1). Treatment with dapagliflozin induced glycosuria that was accompanied by a 2-fold increase in the urine output compared with vehicle-treated mice (Figure 1, A and B). Measurement of fasting blood glucose showed higher levels in BSA-treated mice given vehicle, compared with control mice. Dapagliflozin maintained glycemia at control levels, while lisinopril did not affect this parameter (Table 1). Urine sodium excretion was reduced in mice with protein overload that were given vehicle, compared with control mice (Figure 1C). Levels were significantly enhanced by dapagliflozin with regard to vehicle, but not by lisinopril (Figure 1C). Serum sodium levels were comparable between the experimental groups (Table 1). Mice with protein overload had systolic blood pressure (SBP) levels similar to control mice. An SBP-lowering effect was observed in both dapagliflozin- and lisinopril-treated groups (Table 1).

Measurement of glomerular filtration rate (GFR) by iohexol plasma clearance in the groups of mice with protein-overload proteinuria did not show differences between the groups (BSA + vehicle $(n=5)$, $10.68 \pm 0.81 ; \mathrm{BSA}+$ dapagliflozin $(n=6), 9.04 \pm 0.45 ; \mathrm{BSA}+$ lisinopril $(n=4), 9.49 \pm 1.12 \mu 1 / \mathrm{min} / \mathrm{g})$.

Dapagliflozin limits renal disease progression in mice with proteinuric nephropathy. Treatment with dapagliflozin significantly limited the increase in urinary protein excretion of BSA mice, to such an extent that a $63 \%$ reduction in the urinary protein to creatinine ratio, compared with vehicle-treated mice, was observed (Figure 1D). Consistent with previous data (19), lisinopril treatment resulted in a $72 \%$ reduction in proteinuria (Figure 1D). The antiproteinuric effect of dapagliflozin was associated with amelioration of glomerular lesions - including mesangial matrix expansion, glomerular capillary dilation, and adhesions of the glomerular tuft to the Bowman's capsule - compared with vehicle-treated mice (Figure 2A). The renoprotective effect achieved by SGLT2 inhibition was remarkable, as indicated by the comparison with the ACE inhibitor's effects (Figure 2A). Furthermore, dapagliflozin limited macrophage infiltration in the glomeruli and the renal interstitium of mice injected with BSA, an effect comparable to that obtained after lisinopril treatment (Figure 2B). Tubular damage in mice with 
A

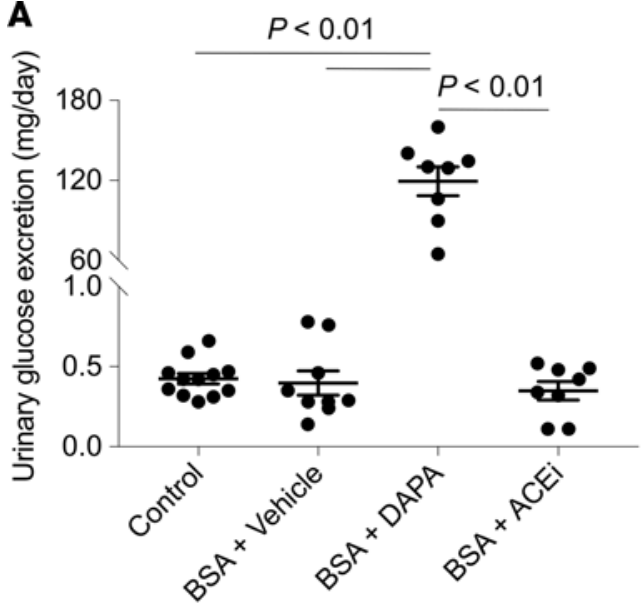

C

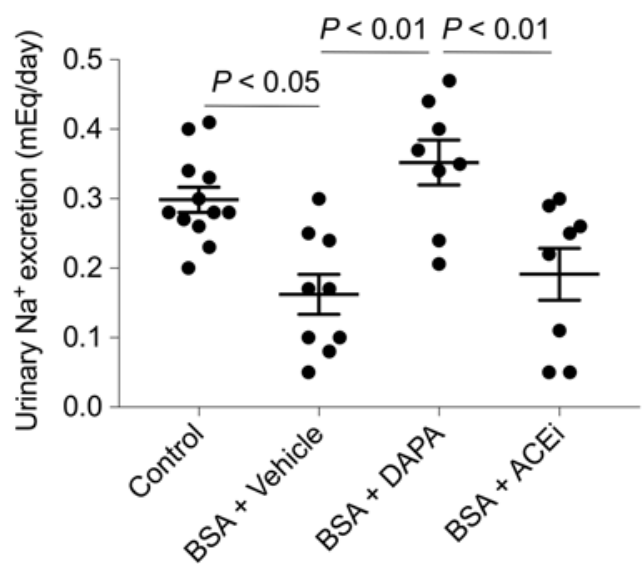

B

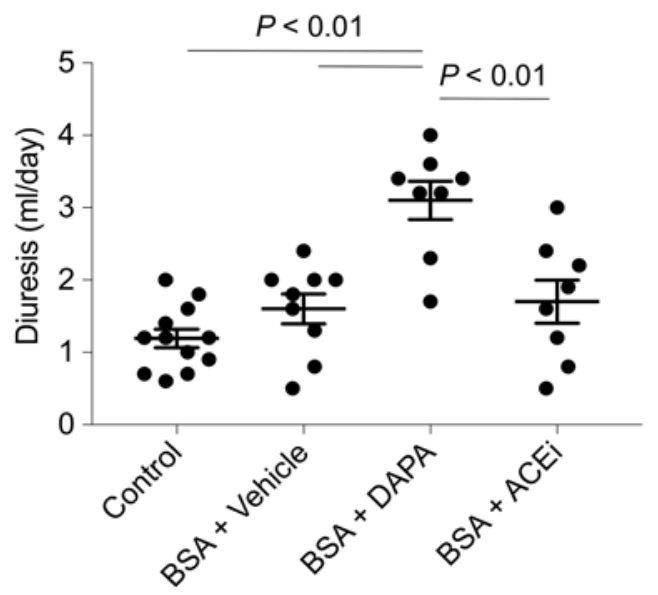

D $\quad P<0.01 P<0.05$

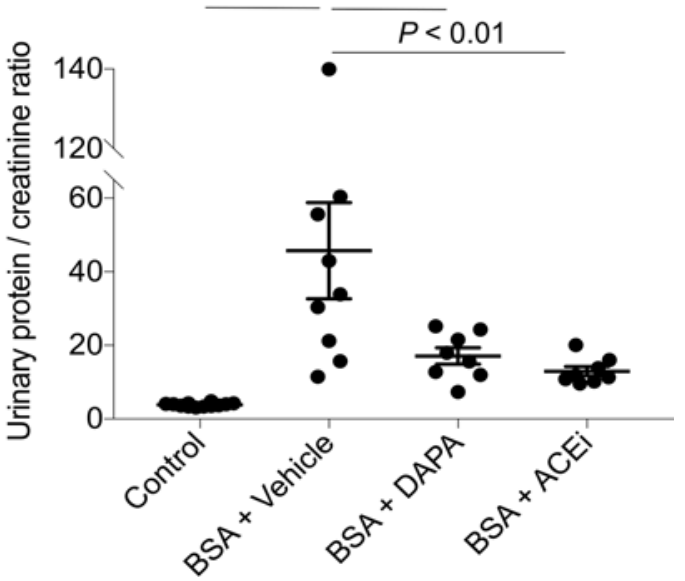

Figure 1. Dapagliflozin induces glycosuria and natriuresis, and limits proteinuria in mice with protein overload. (A-D) Mice were placed in metabolic cages and 24-hour urine was collected for evaluation of glucose excretion (A, $n=8-12)$, diuresis (B, $n=8-12)$, sodium excretion (C, $n=8-12)$, and urinary protein to creatinine ratio $(\mathbf{D}, n=8-12)$ on day 23 after starting BSA injections. Data are the mean \pm SEM and were analyzed by ANOVA with Tukey's post hoc test. DAPA, dapagliflozin; ACEi, ACE inhibitor.

protein-overload proteinuria at this phase of the disease (i.e., 23 days after starting BSA injections) was mild or absent (data not shown).

Dapagliflozin limits podocyte dysfunction and loss in mice with proteinuric nephropathy. On the basis of the antiproteinuric effect observed in dapagliflozin-treated mice, we investigated the drug's possible effects on podocyte damage and loss. Ultrastructural analysis showed intact foot processes of podocytes in control mouse kidney. In glomerular capillaries of mice injected with BSA, focal areas of podocyte damage with effacement of foot processes were seen (Figure 3). These ultrastructural changes were infrequent in mice treated with dapagliflozin or ACE inhibitor (Figure 3).

We evaluated the expression of nephrin, a slit diaphragm protein that maintains slit pore integrity and renal filtration capacity. In mice with protein overload given vehicle, a reduction in the signal intensity of nephrin was observed, compared with control mice (Figure 4). Both treatments ameliorated the defective nephrin expression, although this effect was more evident with dapagliflozin (Figure 4).

In mice injected with BSA, a significant decrease in the number of podocytes labeled for the specific marker Wilms' tumor 1 (WT1) was found, compared with control mice. Podocyte depletion was limited by both dapagliflozin and lisinopril (Figure 5A). The evaluation of podocyte density (Figure 5B), calculated to correct podocyte number for changes in glomerular volume (Figure 5C), showed the remarkable protective effect of dapagliflozin. These results were confirmed by staining for nestin, another podocyte marker (Figure 6). Indeed, the reduction in nestin expression observed in the glomeruli of mice with protein-overload proteinuria given vehicle was significantly reduced by treatments. 
A Control

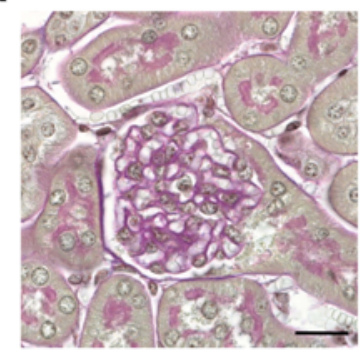

$B S A+D A P A$

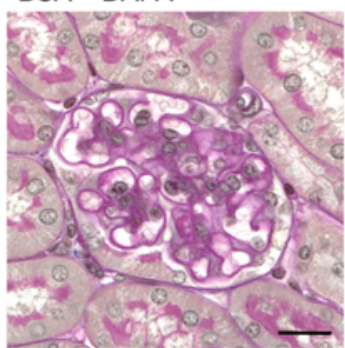

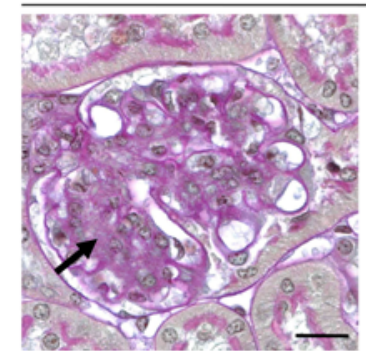

$\mathrm{BSA}+\mathrm{ACEi}$

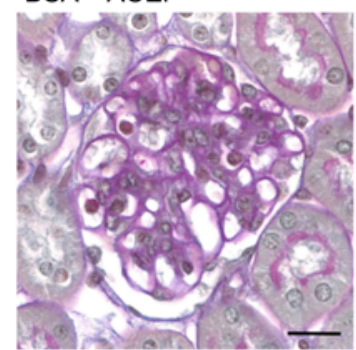

BSA + Vehicle
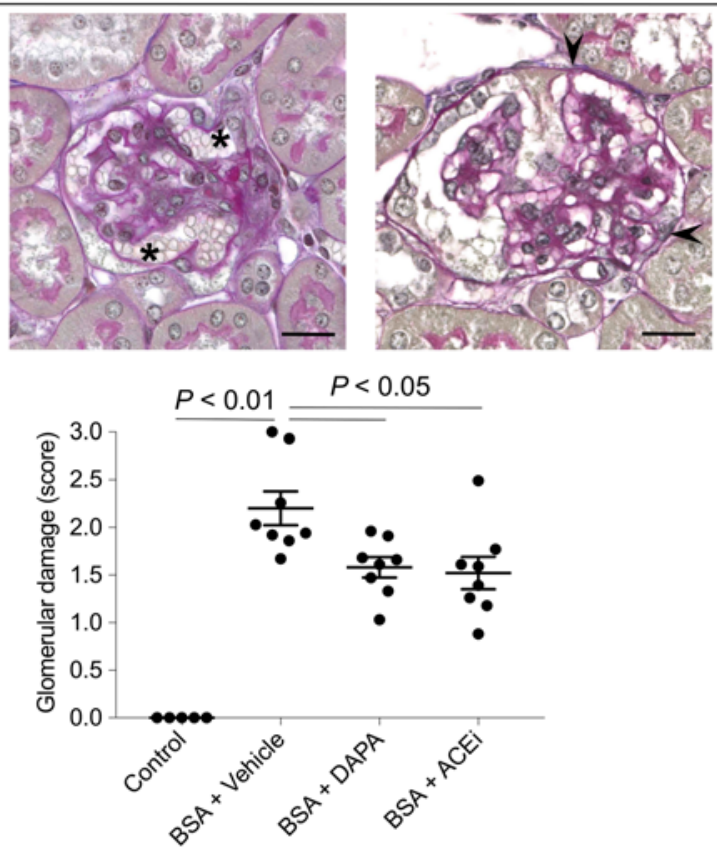

B
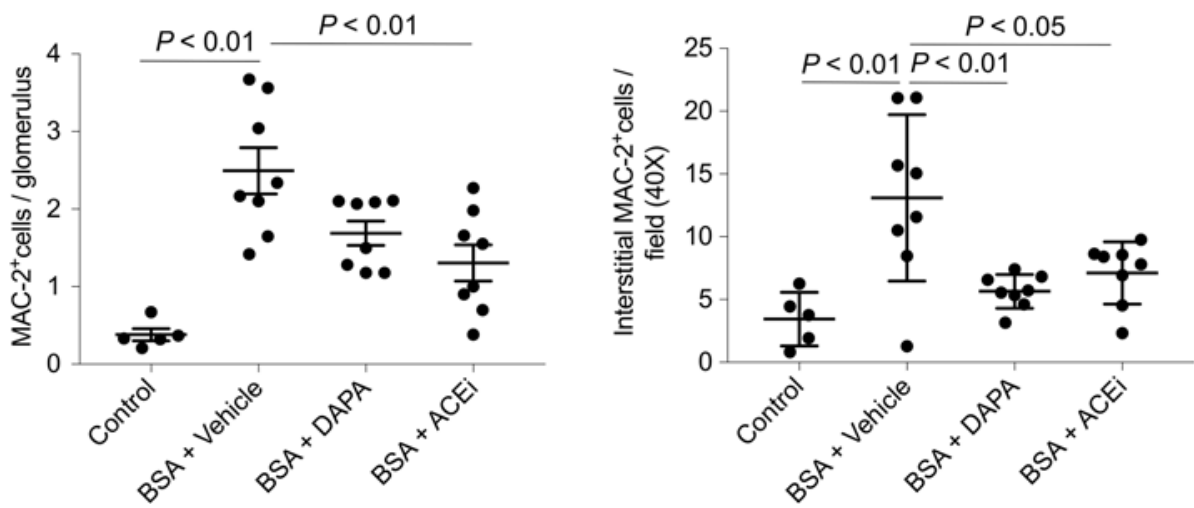

Figure 2. Dapagliflozin limits glomerular structural lesions and inflammation in mice with protein overload. (A) Periodic acid-Schiff-stained images of representative glomeruli from control mice $(n=5)$ and in BSA-mice treated with vehicle, dapagliflozin (DAPA), or ACE inhibitor (ACEi) ( $n=8$ each group). The overall glomerular damage score was calculated by summing mesangial matrix expansion (arrow), glomerular capillary dilation (asterisks), and adhesions of the glomerular tuft to the Bowman's capsule (arrowheads). Scale bars: $20 \mu \mathrm{m}$. (B) Glomerular and interstitial accumulation of Mac-2-positive monocytes/macrophages in control mice $(n=5)$ and in BSA-mice treated with vehicle, dapagliflozin, or ACE inhibitor $(n=8$ each group). Data are the mean \pm SEM and were analyzed by ANOVA with Tukey's post hoc test.

SGLT2 is upregulated in podocytes from mice with protein-overload proteinuria. To explore the possibility that dapagliflozin could have a direct protective effect on podocytes, we assessed whether podocytes express SGLT2. Analysis of kidney sections of control mice showed weak glomerular staining for SGLT2 in a punctate pattern (Figure 7). On the other hand, in mice with protein-overload proteinuria a more intense signal in glomeruli was observed (Figure 7), which localized to podocytes, as shown by costaining of SGLT2 protein with the podocyte marker nephrin. A marked SGLT2 signal was detected in the apical border of the proximal tubule epithelium of control mice, which was not modulated by BSA injections.

SGLT2 protein is expressed by podocytes in culture and upregulated by albumin. Having established that podocytes of mice with protein-overload proteinuria express SGLT2, we moved to an in vitro setting with podocytes loaded with human serum albumin, to investigate whether SGLT2 inhibition could directly counteract the proteinuria-induced podocyte loss we saw in the in vivo model, and to identify possible mechanisms underlying this effect.

First, we wanted to confirm that, as in the in vivo setting, podocytes in culture also express SGLT2, and we studied human podocytes in a resting condition (control) or exposed to albumin overload (6 hours). 


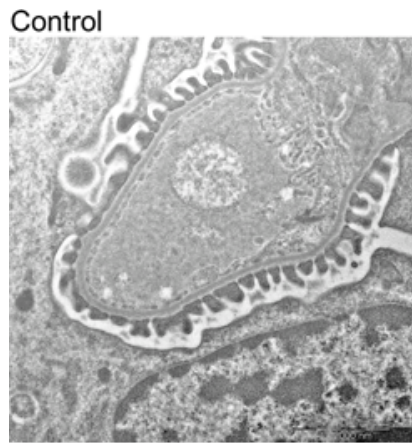

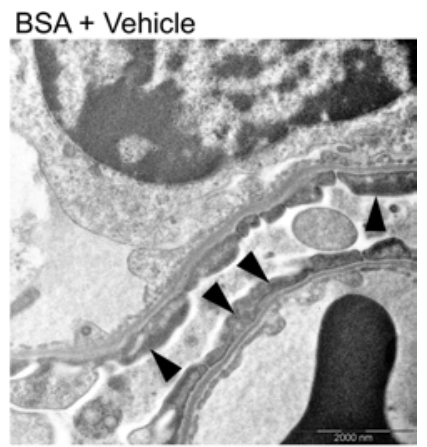

Figure 3. Dapagliflozin limits ultrastructural podocyte damage in mice with protein overload. Representative electron micrographs of glomeruli from control mouse and BSA-mice treated with vehicle, dapagliflozin (DAPA), or ACE inhibitor (ACEi). Focal areas of podocyte damage with effacement of foot processes are indicated by arrowheads in a mouse treated with BSA + vehicle. Scale bars: 2,000 nm.
$\mathrm{BSA}+\mathrm{DAPA}$

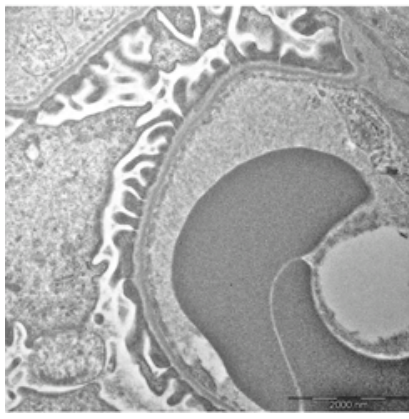

$\mathrm{BSA}+\mathrm{ACEi}$

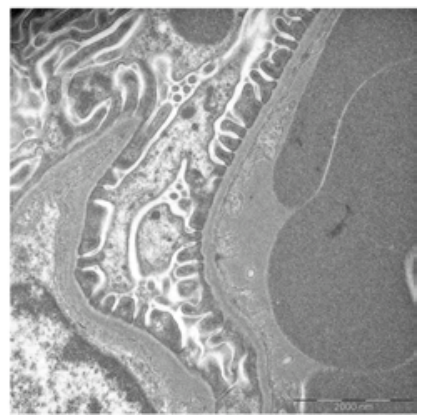

Human renal proximal tubular cells (RPTECs) were used as the positive control (Figure 8A). Western blot analysis of podocyte protein lysates showed the presence of 2 immunoreactive bands with different mobility corresponding to SGLT2. The challenge of podocytes with albumin caused a 3.8-fold increase of SGLT2 protein at 6 hours, compared with control cells, as indicated by the densitometric analysis (Figure $8 \mathrm{~A})$. Upregulation of the SGLT2 protein in response to albumin was preceded at 3 hours by a significant increase of SGLT2 mRNA expression (Figure 8B). Through immunofluorescence experiments we confirmed that podocytes constitutively expressed SGLT2 protein that was distributed on the apical surface of podocytes (nonpermeabilized cells) in a punctate pattern (Supplemental Figure 1; supplemental material available online with this article; https://doi.org/10.1172/jci.insight.98720DS1).

To validate the specificity of the SGLT2 signal, Western blot and immunofluorescence analyses were repeated in albumin-loaded podocytes with the SGLT2 gene silenced by a specific siRNA. A 70\% inhibition of SGLT2 gene expression was achieved in the silenced podocytes (Supplemental Figure 2A). Western blot and immunofluorescence experiments showed that SGLT2 gene silencing translated into reduced SGLT2 protein expression (Supplemental Figure 2, B and C).

Dapagliflozin preserves $F$-actin and $\alpha$-actinin-4 distribution and $\beta 1$-integrin levels in cultured podocytes exposed to albumin. Podocytes possess a complex contractile structure composed of F-actin and associated proteins connected to the glomerular basement membrane (GBM) at focal contacts via integrins, which enable podocytes to efficiently adhere to the GBM and to withstand the oscillating high filtration pressures within the glomerulus $(20,21)$. We previously showed in cultured podocytes that albumin overload caused actin cytoskeleton reorganization associated with the activation of intracellular pathways implicated in glomerular permselectivity dysfunction (22). We therefore assessed the effect of dapagliflozin on podocyte F-actin reorganization in response to albumin. Confluent untreated podocytes (control) exhibited a pattern of F-actin filaments distributed as stress fiber-like bundles along the axis of the cells, as visualized by confocal microscopy (Figure 9A). Albumin induced a marked redistribution of F-actin fibers toward the periphery of the cells, which was counteracted by the SGLT2 inhibitor. As depicted in Figure 9A, dapagliflozin significantly reduced F-actin remodeling induced by 6-hour albumin exposure. Dapagliflozin alone did not induce cytoskeletal redistribution (percentage F-actin rearrangement, $25.7 \% \pm 0.8 \%$ versus control, $24.3 \% \pm 1.2 \%$ ). Considering the effect of dapagliflozin on preventing $\mathrm{F}$-actin alterations induced by albumin, we extended our research to cytoskeleton-associated proteins such as $\alpha$-actinin- 4 , an important actin cross-linking protein that, by interacting with integrins, stabilizes podocytes and regulates cell adhesion and motility $(23,24)$. Control podocytes showed high 
Control

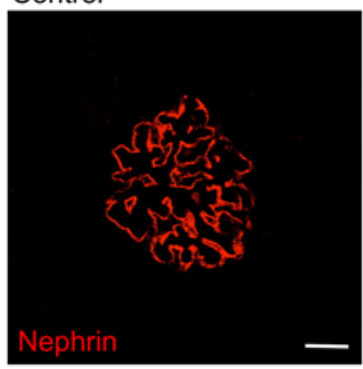

$\mathrm{BSA}+\mathrm{DAPA}$

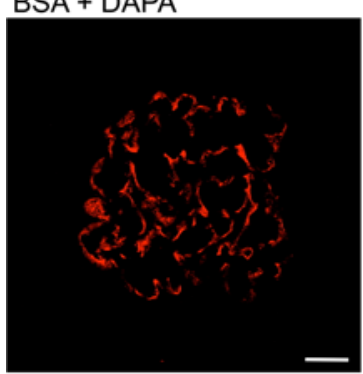

$\mathrm{BSA}+$ Vehicle

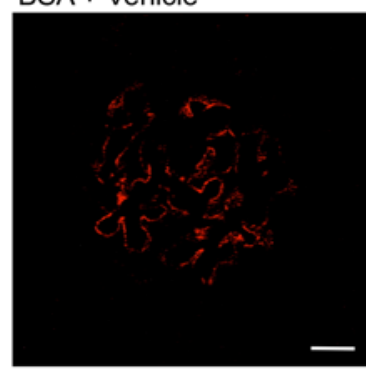

$\mathrm{BSA}+\mathrm{ACEi}$

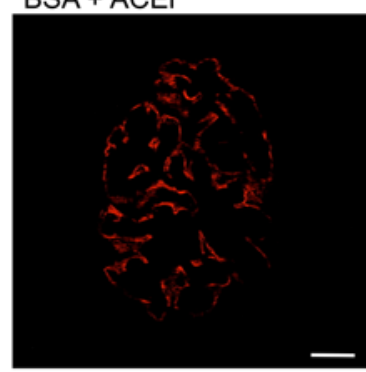

Figure 4. Effects of dapagliflozin on nephrin expression in mice with protein overload. Representative images and quantification showing glomerular nephrin expression (red) in control mice $(n=5)$ and in BSA-mice treated with vehicle, dapagliflozin (DAPA), or ACE inhibitor (ACEi) ( $n=8$ each group), evaluated on day 23 after starting BSA. Scale bars: $20 \mu \mathrm{m}$. Data are the mean \pm SEM and were analyzed by ANOVA with Tukey's post hoc test.

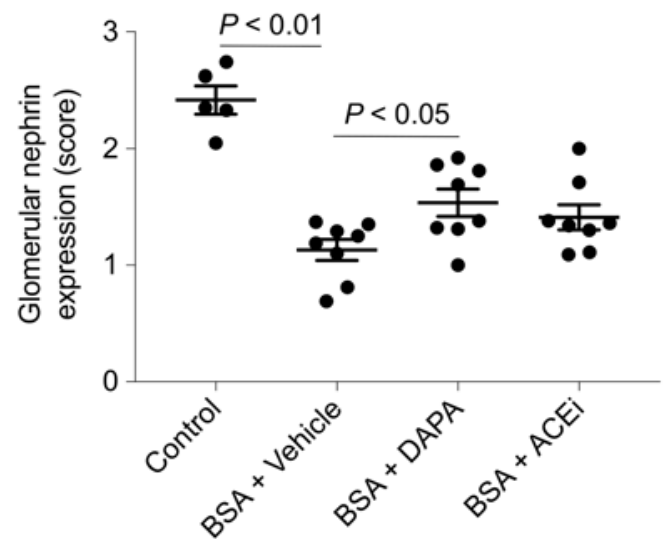

levels of $\alpha$-actinin- 4 that mainly localized along the F-actin stress fibers throughout the cell body, but that was also present within the cortical actin network at the cell periphery (Figure 9B). The exposure to albumin for 6 hours induced cytoplasmic relocalization of $\alpha$-actinin- 4 from a filamentous distribution to a more granular diffuse staining, as also demonstrated by the reduced percentage of podocytes with $\alpha$-actinin- 4 along the F-actin stress fibers compared with control podocytes (Figure 9B). The addition of dapagliflozin limited albumin-induced $\alpha$-actinin- 4 remodeling to a significant extent (Figure 9B). Finally, we evaluated the effect of dapagliflozin on $\beta 1$-integrin expression levels in podocytes exposed to albumin (Figure 9C), and we found that SGLT2 inhibition was effective in limiting the loss of $\beta 1$-integrin in podocytes.

$N F-\kappa B$ mediates albumin-induced expression of SGLT2 in cultured podocytes. Starting from the evidence that the promoter region of $S G L T 2$ contains putative consensus sequences for the binding sites of nuclear factor (NF)-кB (http://www.gene-regulation.com/pub/databases.html\#tranfac; http://jaspar.cgb.ki.se), whose activity was enhanced by albumin load in cultured podocytes in association with the rearrangement of the cytoskeleton (22), we investigated whether NF- $\mathrm{KB}$ activation was responsible for SGLT2 upregulation in albumin-stimulated podocytes. Podocytes were exposed to albumin in the presence of SN50, a specific $\mathrm{NF}-\mathrm{\kappa B}$ nuclear translocation inhibitor $(25,26)$. We found that treatment with SN50 significantly inhibited the gene expression of $S G L T 2$, indicating the pivotal role of NF- $\mathrm{KB}$ in enhancing SGLT2 expression in podocytes in response to albumin load (Figure 10).

$S G L T 2$ is upregulated in podocytes from patients with CKD. Given our experimental findings on the protective effects of SGLT2 inhibition on podocytes, we investigated glomerular SGLT2 expression in renal tissue 


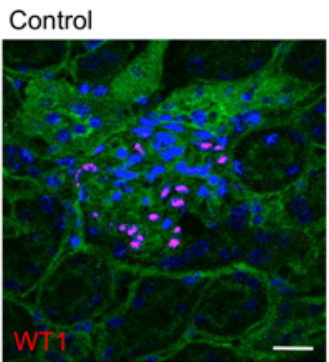

A

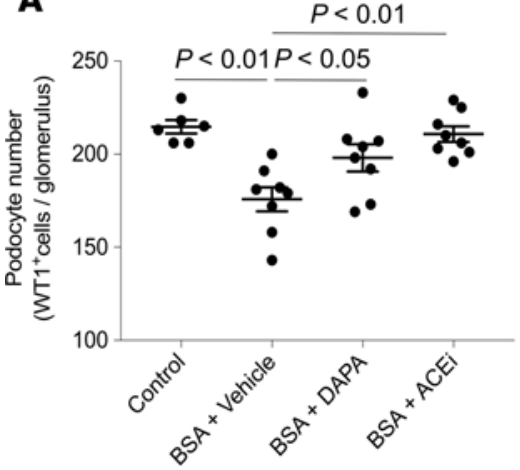

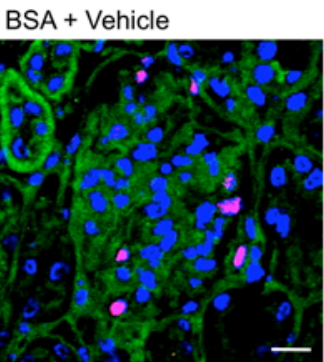

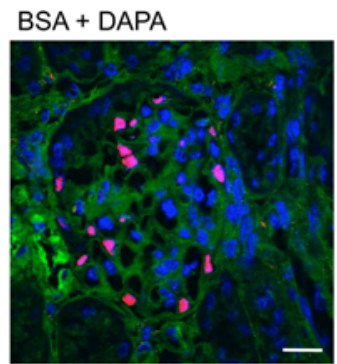

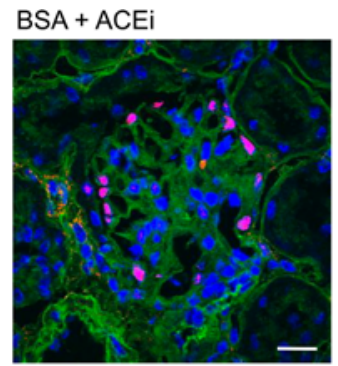

B

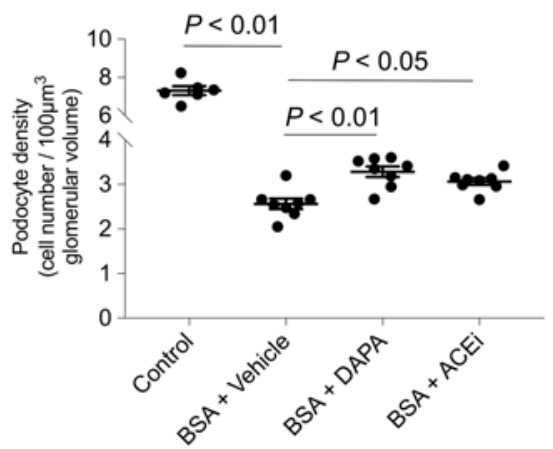

C

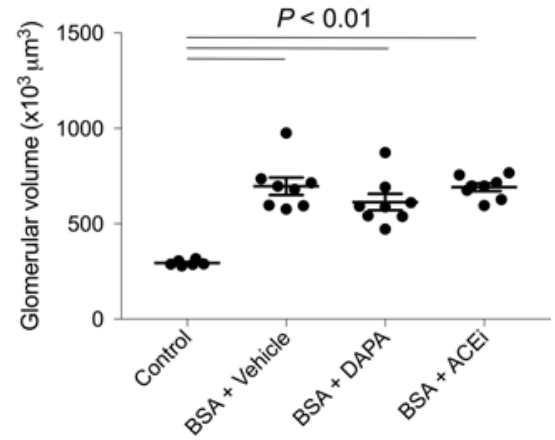

Figure 5. Dapagliflozin limits podocyte depletion in mice with protein-overload proteinuria. Representative images and quantification of WT1-positive podocytes (red) expressed as number per glomerulus (A), podocyte density (B), and glomerular volume (C) in control mice $(n=6)$ and in BSA-mice treated with vehicle, dapagliflozin (DAPA), or ACE inhibitor (ACEi) ( $n=8$ each group), evaluated on day 23 after starting BSA. Scale bars: $20 \mu \mathrm{m}$. Data are the mean \pm SEM and were analyzed by ANOVA with Tukey's post hoc test.

from 4 proteinuric patients with idiopathic membranous nephropathy. Clinical characteristics are outlined in Table 2. A weak SGLT2 staining was observed in glomeruli of control kidneys (i.e., the uninvolved portions collected from tumor nephrectomy). At variance, a more evident staining was found in proteinuric patients, which was localized to podocytes. Representative images are shown in Figure 11.

\section{Discussion}

In this study, we provide experimental evidence that SGLT2 inhibition with dapagliflozin has renoprotective effects in a model of nondiabetic kidney disease. Dapagliflozin markedly reduced proteinuria and glomerular lesions and limited podocyte damage and loss in mice with progressive proteinuric nephropathy induced through repeated BSA injections.

SGLT2 inhibitors are important blood glucose-lowering agents that primarily function by reducing glucose reabsorption via SGLT2 in the proximal tubule. However, SGLT2 inhibition also interferes with nonglycemic pathways, recognized to be implicated in the renopropective benefits recently reported in clinical trials with type 2 diabetic patients (10-12). Indeed, SGLT2 inhibition promotes natriuresis that regulates renal and systemic hemodynamic functions, including lowering blood pressure and glomerular hyperfiltration and plasma volume, and proteinuria (17). The pleiotropic effects of SGLT2 inhibitors would render them an applicable new therapeutic opportunity for nondiabetic CKD, particularly for patients in whom nephroprotection by standard therapy with RAS blockade is only partial (2). To establish the potential of SGLT2 inhibition in proteinuric glomerular disease, we used the mouse model of protein-overload proteinuria in which the loss of the permselective properties of the glomerular barrier to proteins is a major determinant for disease progression. Mice were studied until 23 days after starting BSA injection, a time at which they exhibit proteinuria and glomerular lesions but mild tubular damage. Treatment with dapagliflozin had a remarkable antiproteinuric effect, associated with amelioration of the glomerular lesions, similar to that observed with ACE inhibitor. This is the first piece of evidence, to the best of our knowledge, of the effectiveness of SGLT2 inhibitors in an experimental model of nondiabetic CKD. There is a previous study that demonstrated lack of renoprotective effects of this class of drugs in 5 of 6 nephrectomized rats, a classical model of progressive nondiabetic CKD (27). Treatment with dapagliflozin did not affect hypertension, proteinuria, or GFR, nor did it attenuate the extension of glomerulosclerosis and tubulointerstitial 

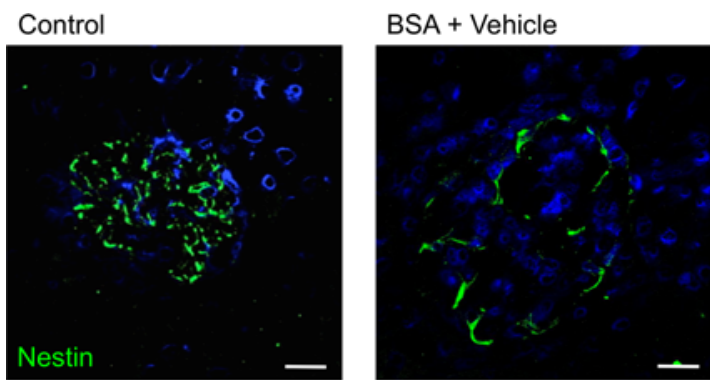

$\mathrm{BSA}+\mathrm{DAPA}$

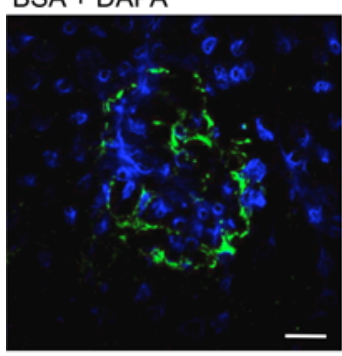

$\mathrm{BSA}+\mathrm{ACEi}$
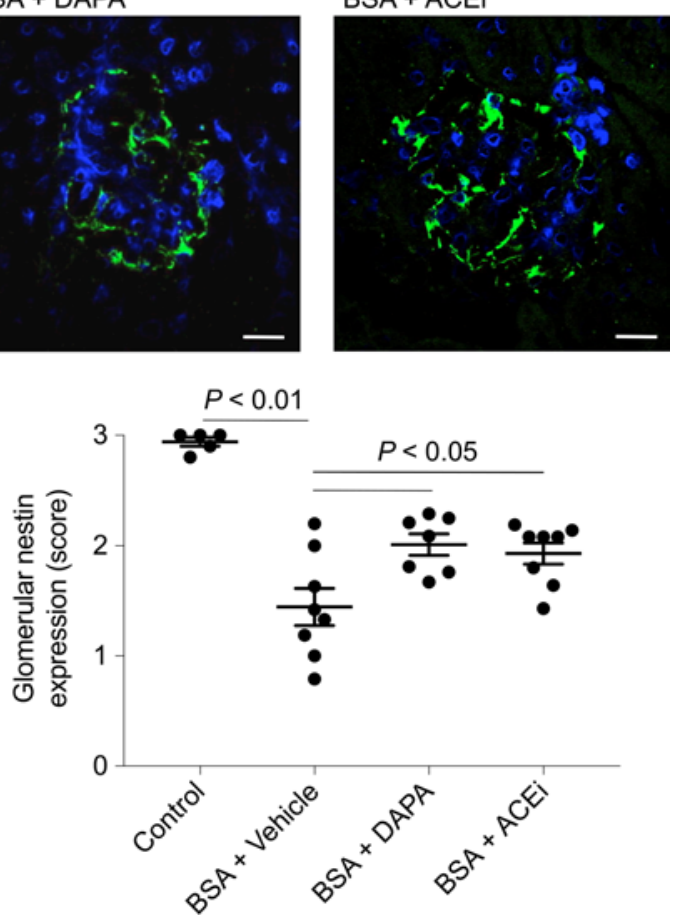

Figure 6. Dapagliflozin ameliorates defective podocyte nestin expression in mice with protein overload. Representative images and quantification showing glomerular nestin expression (green) in control mice $(n=5)$ and in BSA-mice treated with vehicle, dapagliflozin (DAPA), or ACE inhibitor (ACEi) ( $n=7-8$ each group), evaluated on day 23 after starting BSA. Scale bars: $20 \mu \mathrm{m}$. Data are the mean \pm SEM and were analyzed by ANOVA with Tukey's post hoc test.

fibrosis, in the face of substantial glycosuria (27). It has to be pointed out that in the remnant rat kidney the expression of SGLT2 was markedly decreased compared with the control kidneys (28), suggesting that SGLT2 may not be implicated in the disease progression of this severe CKD model and that further inhibition of SGLT2 by dapagliflozin could even be detrimental. Indeed, pharmacologically induced prolonged and sustained glycosuria with SGLT2 inhibitors may raise the risk of developing genital infections and, to a lesser extent, urinary tract infections $(29,30)$.

Our data indicate that SGLT2 plays a role in the development of renal disease of mice with proteinoverload proteinuria. Thus, SGLT2 was highly expressed in the kidneys of mice after BSA injections and importantly, SGLT2 inhibition prevented the increase in blood glucose levels and corrected defective natriuresis in mice with protein-overload proteinuria. However, the natriuretic effect of dapagliflozin was not associated with changes in GFR, as we measured at the end of the study on day 23 after starting BSA injections, suggesting that in our experimental setting, amelioration of proteinuria and glomerular lesions was unlikely the consequence of natriuresis-dependent glomerular hemodynamic function.

Following the important finding that in mice with protein-overload proteinuria dapagliflozin limited podocyte damage and loss, which is a critical event in determining proteinuria and progression of the glomerular disease, we focused on podocytes. We provide the observation that mouse podocytes expressed SGLT2, as shown by the colocalization of SGLT2 and nephrin staining, and that levels increased after BSA injections. Notably, SGLT2 was expressed in podocytes of proteinuric CKD patients, and the staining intensity was higher than that found in control subjects. A direct effect of protein overload in modulating SGLT2 expression in podocytes derives from our in vitro experiments in cultured human podocytes exposed to a high albumin concentration. That a sodium-dependent glucose cotransporter 

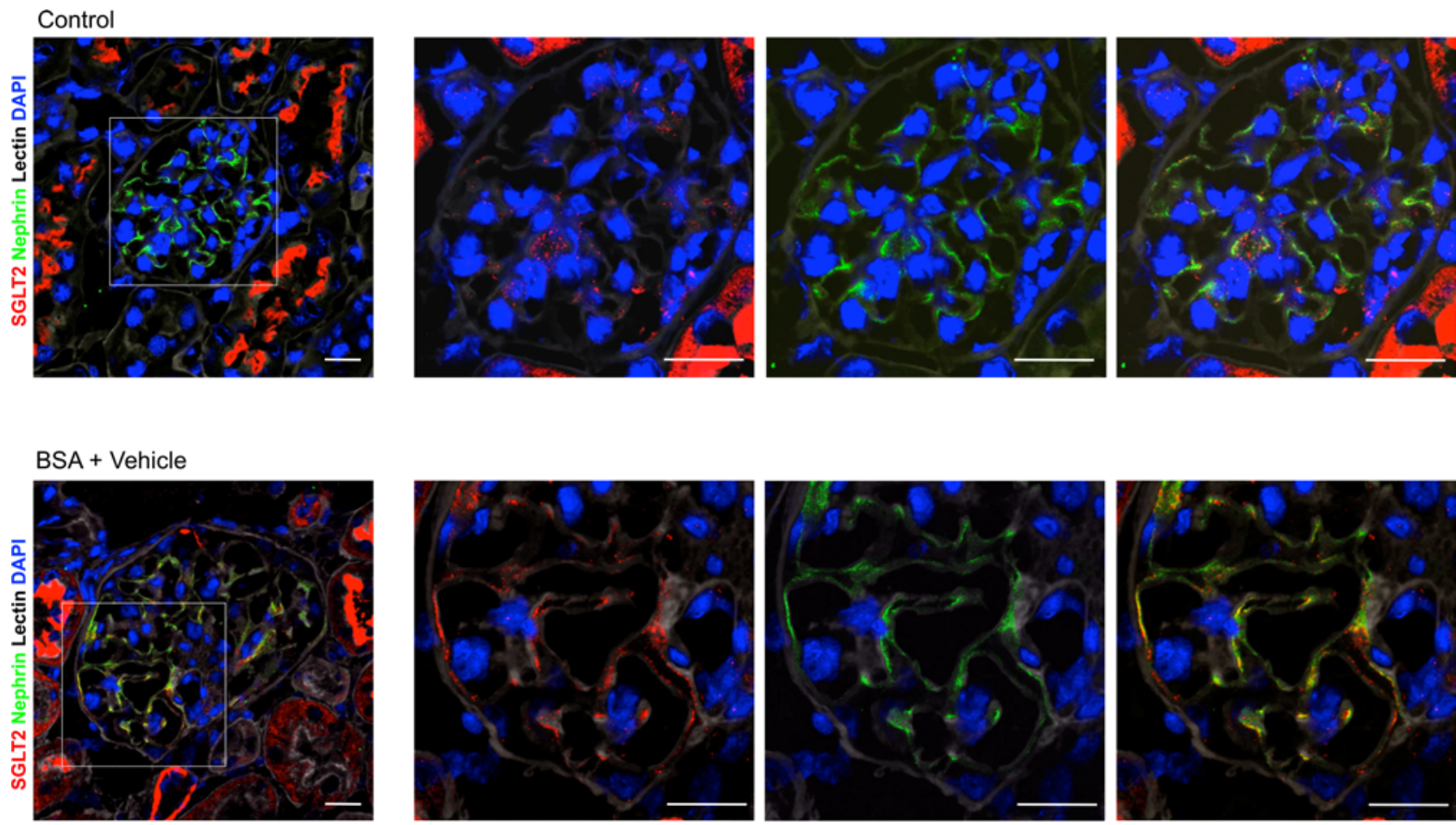

Figure 7. SGLT2 is expressed in podocytes and upregulated after BSA injections. Representative images of renal tissue from control mice and mice treated with BSA showing merged area (yellow) of costaining of glomerular SGLT2 expression (red) and nephrin (green) in podocytes. Boxes indicate area of enlarged images to the right. Nuclei and cell membranes are stained with DAPI (blue) and Cy5-labeled lectin (gray), respectively. Scale bars: $20 \mu \mathrm{m}$.

could be expressed in podocytes was previously suggested by the observation that phlorizin, a potent competitive inhibitor of all SGLT forms, decreased glucose uptake in cultured rat podocytes (31). It is widely accepted that podocytes have a glucose (GLUT) transporter system that includes GLUT1, GLUT2, and GLUT4 (31-34). However, the observation that podocytes, when exposed to a mechanical stress, exhibited enhanced glucose uptake but decreased expression of GLUTs, either under normal or high glucose conditions, suggested that stress/injury modulated the activity of glucose transporters other than GLUTs in podocytes (35). Translating this observation to our in vitro setting, podocytes stressed by albumin overload showed a marked remodeling of F-actin and $\alpha$-actinin- 4 filaments, and loss of $\beta 1$-integrin together with increased SGLT2 mRNA and protein expression on their surface. Interestingly, the promoter region of SGLT2 contains putative consensus sequences for the binding sites of $\mathrm{NF}-\kappa \mathrm{B}$. We previously showed that albumin load caused NF- $\mathrm{B}$ activation in cultured podocytes in association with the rearrangement of the cytoskeleton (22). In particular, the abnormal uptake of albumin caused Rho kinase-dependent F-actin cytoskeletal rearrangement that translated into the activation of the integrin-associated nonreceptor tyrosine kinase focal adhesion kinase (FAK), in turn responsible for $\mathrm{NF}-\kappa \mathrm{B}$-dependent upregulation of genes implicated in glomerular permselective dysfunction and proteinuria (22). Through experiments with a specific NF- $\mathrm{B}$ inhibitor here we provide evidence that NF- $\mathrm{B}$ activation in response to albumin load leads to the upregulation of SGLT2, which in turn contributes to structural and functional changes in podocytes. In support of this possibility, indeed we found that SGLT2 inhibition with dapagliflozin through a direct effect on podocytes ameliorates albumin-induced cytoskeletal rearrangement. Several studies have demonstrated that in conditions of high intracellular glucose, podocytes change their size and shape by reorganizing the actin cytoskeleton to cover the portion of the basement membrane left naked by lost podocytes (36-39). It is conceivable that the upregulation of SGLT2 expression in podocytes induced by albumin may increase intracellular glucose uptake, thus contributing to cytoskeletal rearrangement.

To summarize, we have demonstrated for the first time to our knowledge that (a) SGLT2 inhibition with dapagliflozin provides renoprotection in a model of nondiabetic kidney disease to a similar extent 
A

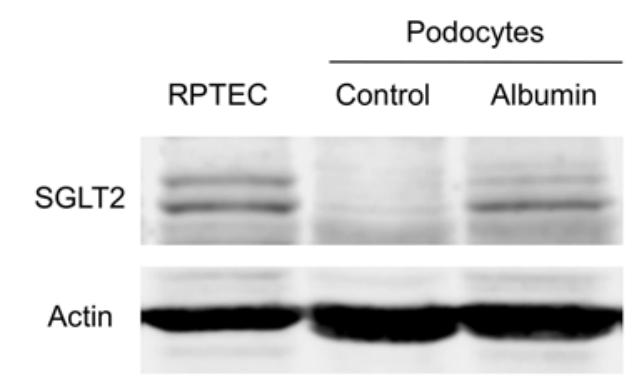

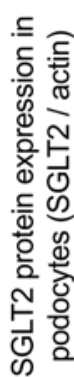

B
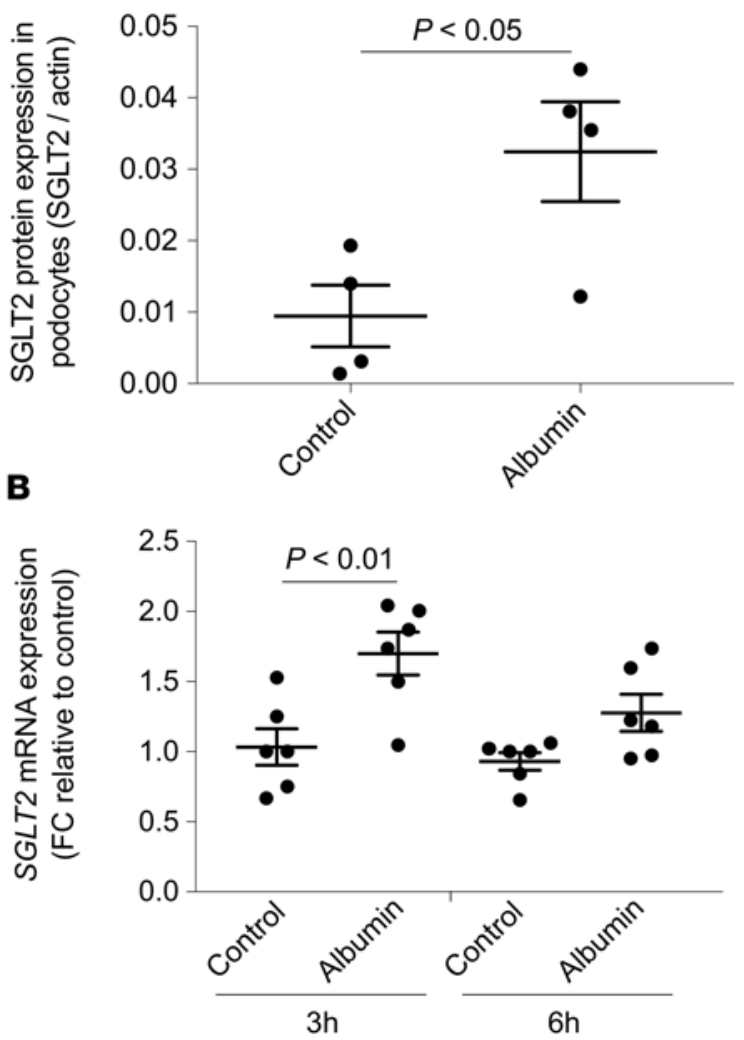

Figure 8. Human cultured podocytes express SCLT2 that is upregulated by albumin. (A) Representative Western blot and densitometric analysis of SGLT2 protein in renal proximal tubular cells (RPTECs), used as positive control, and podocytes exposed to medium alone (control) or albumin ( $10 \mathrm{mg} / \mathrm{ml}, 6$ hours). Actin was used as sample loading control. Data are the mean \pm SEM ( $n=4$ samples) and were analyzed by Student's $t$ test. (B) SCLT2 mRNA expression evaluated by real-time qPCR analysis in podocytes exposed to medium alone (control) or albumin $(10 \mathrm{mg} / \mathrm{ml})$ for 3 and 6 hours. Hypoxanthine phosphoribosyltransferase 1 (HRPT1) was used as endogenous control. SCLT2 levels were normalized to HPRT1 levels and reported as fold change (FC) relative to the corresponding control. Data are the mean \pm SEM ( $n=6$ samples) and were analyzed by ANOVA with Tukey's post hoc test.

as an ACE inhibitor does; (b) in mice with protein-overload proteinuria, dapagliflozin reduces proteinuria and ameliorates podocyte dysfunction and loss; (c) podocytes express SGLT2 and levels are enhanced by albumin load both in the in vivo model and in vitro in an NF-kB-dependent manner; and (d) in cultured podocytes dapagliflozin limits cytoskeletal remodeling induced by albumin load.

Our data suggesting that the podocyte is a target of an SGLT2 inhibitor indicate that this class of drugs is a potential therapeutic option for patients with proteinuric glomerular disease, particularly those who do not respond to standard therapy. Further studies are needed to better clarify the mechanisms underlying the protective effect of dapagliflozin on podocytes and to identify the CKD subtypes that may benefit from SGLT2 inhibition.

\section{Methods}

Animal experiments. Protein-overload proteinuria was induced as previously described (18) in male 9-weekold C57BL/6N mice (Charles River Laboratories Italia). Unilateral nephrectomy was performed 5 days before starting injections of BSA (day 0). Mice received i.p. injections of 5 and $10 \mathrm{mg} / \mathrm{g}$ body weight of BSA (A-7906, Sigma-Aldrich) for the first 2 days, then BSA was given at $15 \mathrm{mg} / \mathrm{g}$ body weight 5 days a week for 3 weeks. Control mice received the same volume of saline. Beginning on day 1 of BSA injection, animals were treated from 1 to 23 days with vehicle $(n=9)$, dapagliflozin $(1.5 \mathrm{mg} / \mathrm{kg}$, in the drinking water, provided by AstraZeneca Lab Italia) $(n=8)$, and lisinopril $(40 \mathrm{mg} / 1$ in the drinking water, provided by 
A

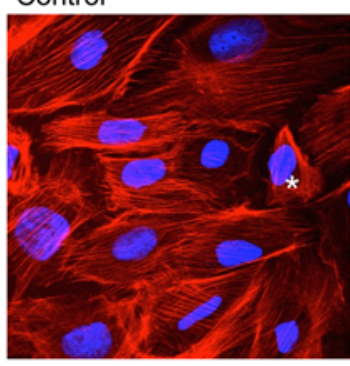

B

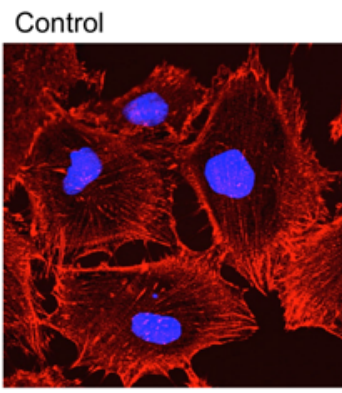

c

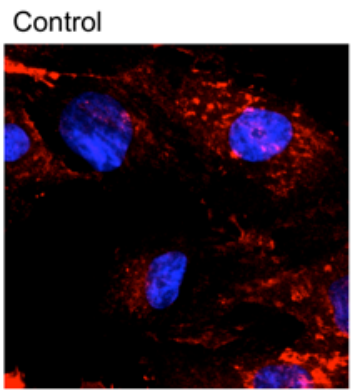

Albumin

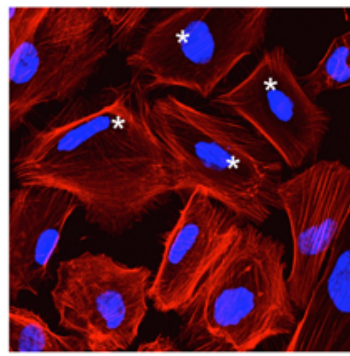

Albumin

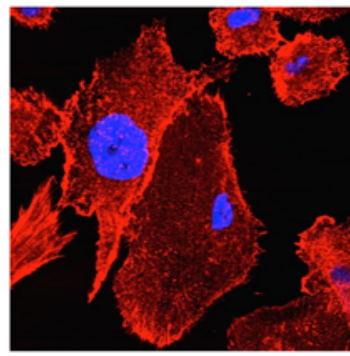

Albumin

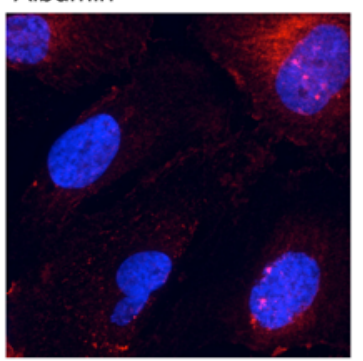

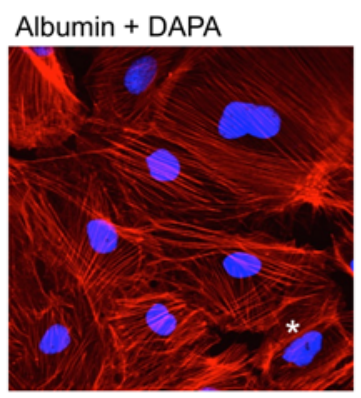
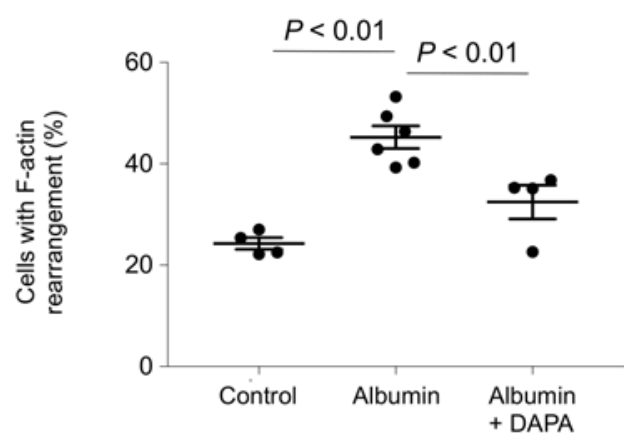

Albumin + DAPA
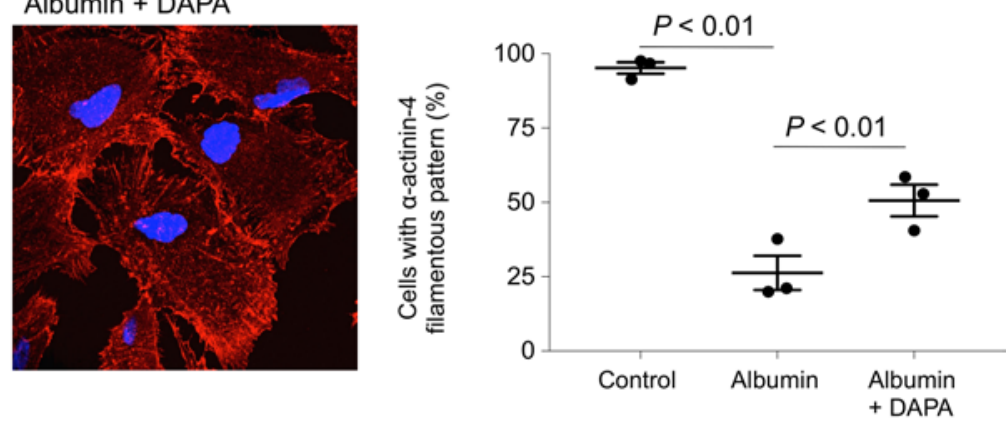

Albumin + DAPA
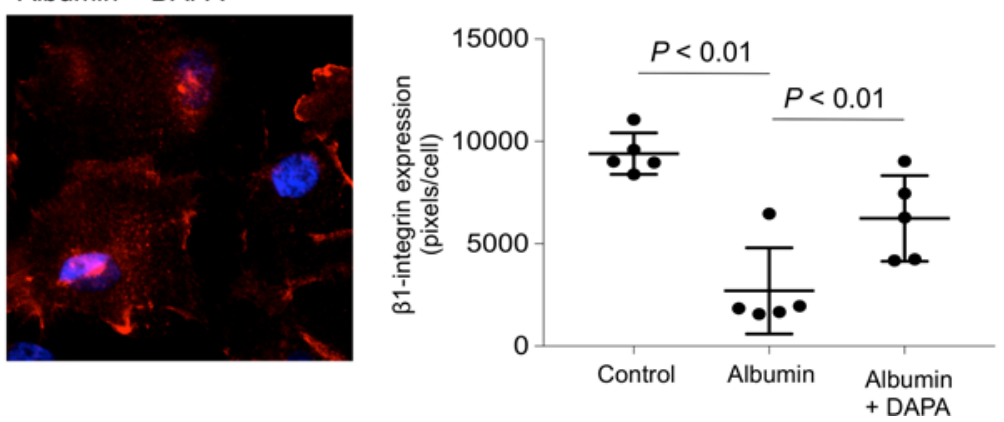

Figure 9. Effect of dapagliflozin on cytoskeletal remodeling in podocytes loaded with albumin. Representative images and quantification of immunofluorescence staining for F-actin (red) (A), $\alpha$-actinin-4 (red) (B), and $\beta 1$-integrin (red) (C) in podocytes exposed to control medium (control) or albumin $(10 \mathrm{mg} / \mathrm{ml})$, in the presence or absence of dapagliflozin (DAPA, $10 \mathrm{nM}$ ) for 6 hours. Nuclei were counterstained with DAPI (blue). Asterisks indicate podocytes with F-actin cytoskeletal remodeling. Quantifications were performed in 15 fields per sample. Data are the mean \pm SEM ( $n$ $=4-6$ samples for F-actin, $n=3$ samples for $\alpha$-actinin- $4, n=5$ samples for $\beta 1$-integrin) and were analyzed by ANOVA with Tukey's post hoc test. Original magnification, $\times 630$.

AstraZeneca Lab Italia) $(n=8)$ as standard therapy for comparison. The group of control mice $(n=12$, group 4) was followed for the same time. Mice were sacrificed on day 23 after starting BSA. All mice were housed in a constant-temperature room with a 12-hour dark/12-hour light cycle in a specific pathogen-free facility, and fed a standard diet.

Biochemical and renal function parameters. Blood glucose was assessed with a reflectance meter (OneTouch UltraEasy, LifeScan). Serum and urinary $\mathrm{Na}^{+}$levels were measured by the autoanalyzer CX5 (Beckman Instruments). Urinary glucose and creatinine levels were measured by Cobas Mira autoanalyzer (Roche Diagnostics Systems). Urinary protein excretion was measured by the Coomassie method using a Cobas Mira autoanalyzer. GFR measurement was performed by iohexol plasma clearance in conscious animals as described previously (40).

SBP. SBP was measured with a computerized tail-cuff system in conscious mice (BP-2000 Blood Pressure Analysis System, Visitech System).

Renal histology. Duboscq-Brazil-fixed paraffin-embedded kidney sections $(3 \mu \mathrm{m})$ were stained with periodic acid-Schiff (PAS) reagent. At least 50 glomeruli were examined for each animal, and the extent of 


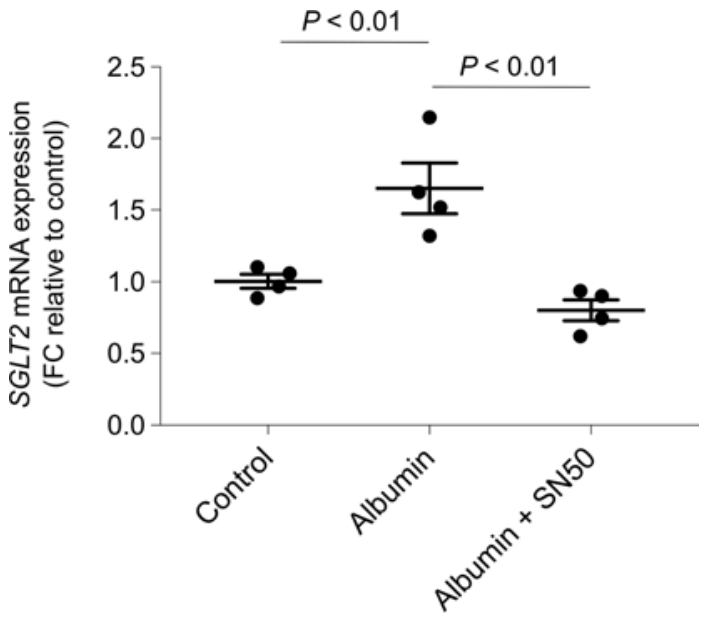

Figure 10. NF-KB mediates albumin-induced SCLT2 mRNA expression in human cultured podocytes. SGLT2 mRNA expression evaluated by real-time qPCR analysis in podocytes exposed to medium alone (control) or albumin $(10 \mathrm{mg} / \mathrm{ml})$ in the presence or absence of SN50 $(10 \mu \mathrm{M})$ for 3 hours. Hypoxanthine phosphoribosyltransferase 1 (HRPT1) was used as endogenous control. SGLT2 levels were normalized to HPRT1 levels and reported as fold change relative to control. Data are the mean \pm SEM ( $n=4$ samples) and were analyzed by ANOVA with Tukey's post hoc test.

lesions was expressed with a cumulative score from 0 to 3 (mild, moderate, and severe) that includes mesangial matrix expansion, glomerular capillary dilation, and adhesions of the glomerular tuft to the Bowman's capsule. All biopsies were reviewed by a blinded pathologist, who was unaware of the nature of the experimental groups. Samples were examined using ApoTome Axio Imager Z2 (Zeiss).

Renal ultrastructural analysis. Fragments of kidney tissue were fixed overnight in $2.5 \%$ glutaraldehyde in $0.1 \mathrm{M}$ cacodylate buffer ( $\mathrm{pH} 7.4$ ) and washed repeatedly in the same buffer. After postfixation in $1 \% \mathrm{OsO}_{4}$, specimens were dehydrated through ascending grades of alcohol and embedded in Epon resin. Ultrathin sections were stained with uranyl acetate replacement (UAR, Electron Microscopy Sciences) and lead citrate and examined using transmission electron microscopy (Morgagni 268D, Philips).

Glomerular podocyte count. Podocytes were identified as cells positive for WT1. OCT-frozen kidney sections were incubated overnight with rabbit anti-WT1 antibody (1:400, C-19, Santa Cruz Biotechnology), followed by Cy3-conjugated goat anti-rabbit IgG (1:100, 111-165-144, Jackson ImmunoResearch Laboratories). Nuclei and cell membranes were stained with DAPI (Sigma-Aldrich) and FITC-WGA lectin (Vector Laboratories). At least 30 glomeruli/section for each animal were randomly acquired using confocal laser scanning microscopy. The estimation of the average number of podocytes per glomerulus was determined by morphometric analysis as previously described (41).

Immunohistochemical analyses in renal tissue. For Mac-2 evaluation, Duboscq-Brazil-fixed, 3- $\mu$ m paraffinembedded kidney sections were used. Sections were incubated for 5 minutes with Peroxidazed 1 (Biocare Medical) to quench endogenous peroxidase, after antigen retrieval in citrate buffer. After blocking for 30 minutes with Rodent Block M (Biocare Medical), sections were incubated overnight at $4^{\circ} \mathrm{C}$ with rat anti-Mac-2 (1:600, M3/38, Cedarlane), followed by biotinylated goat anti-rat antibody (1:200, R40015, Caltag) and avidin/biotin complex (Vector Laboratories). Slides were counterstained with hematoxylin, and observed using light microscopy (ApoTome Axio Imager Z2). Negative controls were obtained by omitting the primary antibody on adjacent sections. Mac-2-positive monocyte/macrophages within glomeruli were counted in a minimum of 50 glomerular cross sections and expressed as average number of cells per glomerulus. Positive cells

Table 2. Demographic and clinical characteristics of patients with idiopathic membranous nephropathy

\begin{tabular}{lc}
\hline Patients, $n$ & 4 \\
Age, years-range & $42 \pm 2$ \\
Sex, Male/Female & $2: 2$ \\
SBP, mmHg & $121.25 \pm 8.26$ \\
DBP, mmHg & $72.50 \pm 4.79$ \\
Proteinuria, g/day & $3.12 \pm 0.14$ \\
Serum creatinine, mg/dl & $0.86 \pm 0.13$
\end{tabular}

Data are expressed as the mean \pm SEM. SBP, systolic blood pressure; DBP, diastolic blood pressure. 
A

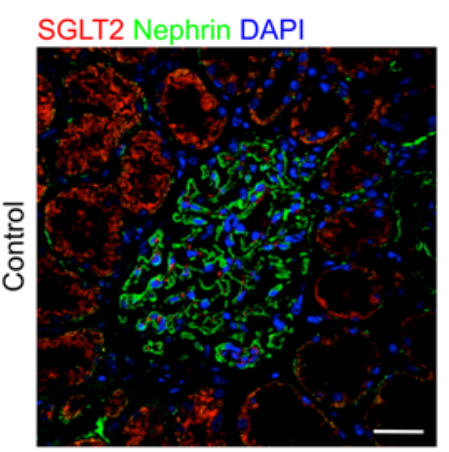

B

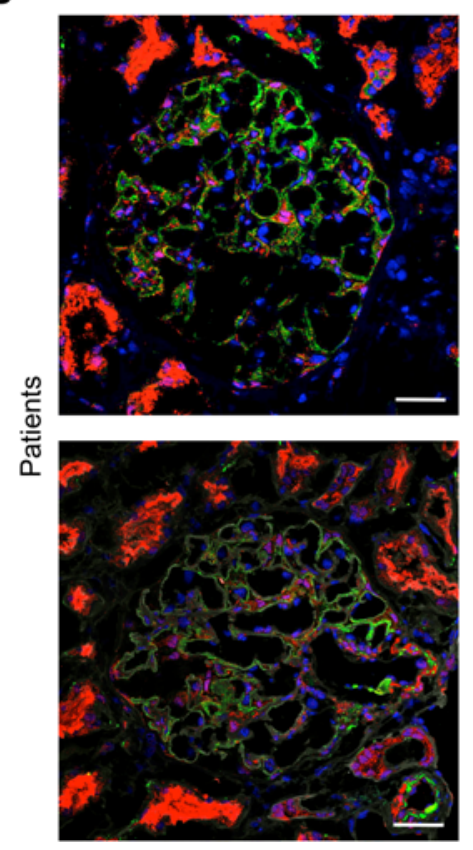

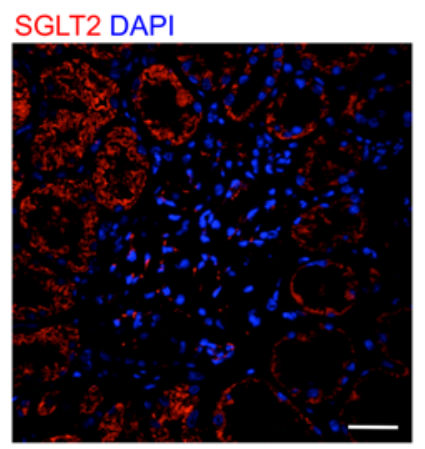
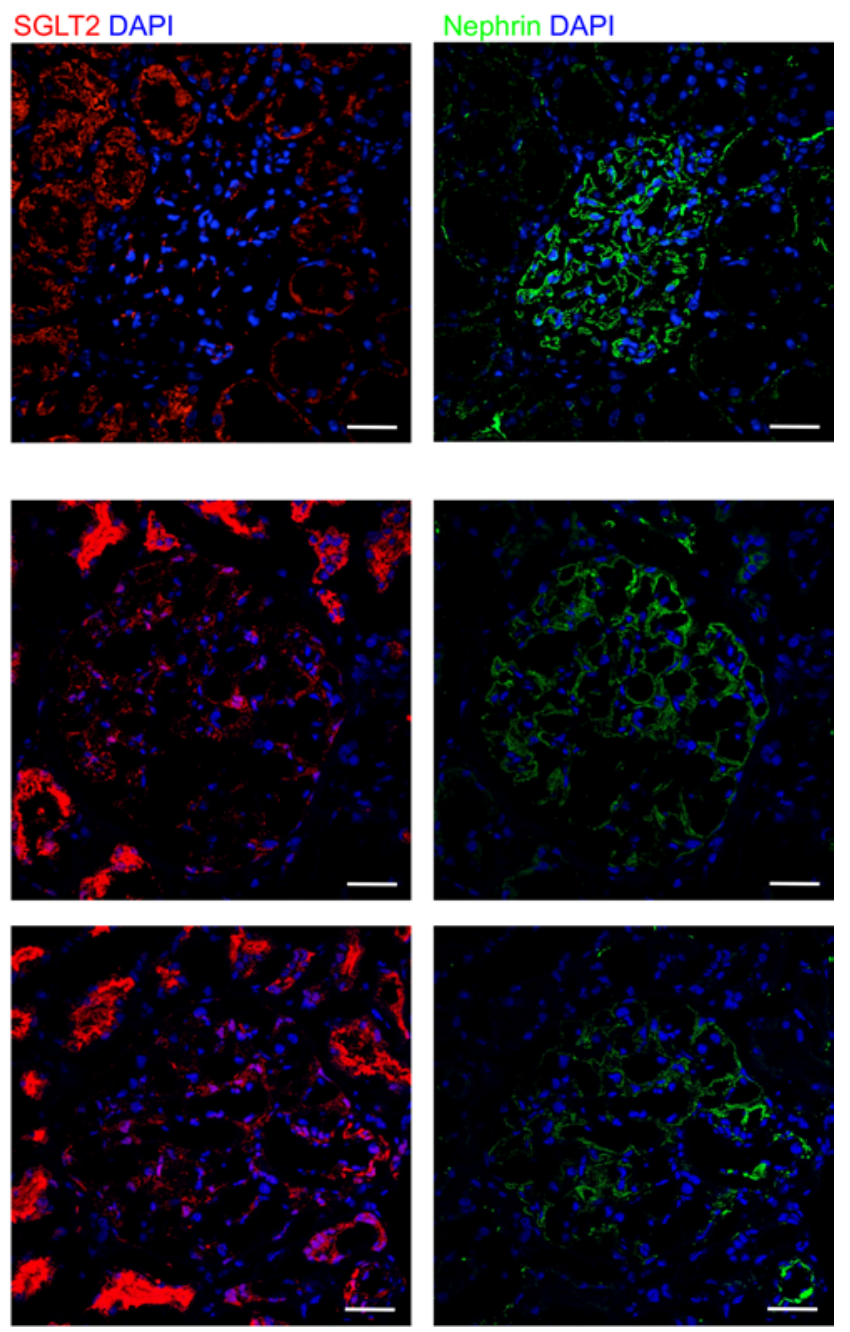

Figure 11. SGLT2 expression in renal biopsies of patients with idiopathic membranous nephropathy. (A) Representative double immunofluorescence staining for SGLT2 (red) and nephrin (green) in normal control kidney $(n=$ 3 ) and (B) in the renal biopsy of proteinuric patients with idiopathic membranous nephropathy $(n=4)$. DAPI (blue) stains nuclei. Scale bars: $20 \mu \mathrm{m}$.

were counted in at least 15-20 randomly selected interstitial high-power fields (HPFs; $\times 400)$ for each animal. The immunohistochemical analyses and scoring assays were done by individuals unaware of sample identity.

OCT-frozen kidney sections were incubated overnight with the following primary antibodies: goat anti-nephrin (1:100, N-20, Santa Cruz Biotechnology Inc.) followed by Cy3-conjugated secondary antibody (1:400, 705-165-147, Jackson ImmunoResearch Laboratories); rat anti-nestin (1:300, 7A3, Abcam) followed by FITC-conjugated secondary antibody for nestin staining (1:200, F6258, Sigma-Aldrich); rabbit anti-SGLT2 (1:25 and 1:400, ab85626, Abcam) followed by Cy3-conjugated secondary antibody (1:50, 711165-152, Jackson ImmunoResearch Laboratories). When double immunostaining for SGLT2 and nephrin was performed, a FITC-conjugated secondary antibody for nephrin staining was used (1:80, 705-096-147, Jackson ImmunoResearch Laboratories). Nuclei and renal structure were stained with DAPI and with Alexa Fluor 633-WGA (Thermo Fisher Scientific), respectively. Negative controls were obtained by omitting the primary antibody on adjacent sections. Samples were examined using an inverted confocal laser microscope (LSM 510 Meta; Zeiss). Nephrin and nestin staining was quantified by score from 0 to 3 (nephrin, $0-0.5$ : absent or weak and fragmented signal, 1 : linear and thin signal, 2: linear signal, 3 : linear and intense signal; nestin, $0-0.5$ : absent or weak staining in a few podocytes, 1 : moderate staining in podocytes with altered distribution, 2: moderate podocyte staining, 3: intense podocyte staining). At least 15-20 glomeruli/section for each animal were randomly analyzed. 
For immunostaining in human biopsies, OCT-frozen kidney sections were incubated with goat antinephrin (1:50, N-20, Santa Cruz Biotechnology Inc.) and rabbit anti-SGLT2 (1:100, ab85626, Abcam) antibodies, followed by FITC-conjugated (1:80, 705-096-147, Jackson ImmunoResearch Laboratories) or Cy3conjugated secondary antibodies (1:50, 711-165-152, Jackson ImmunoResearch Laboratories), respectively. Nuclei were stained with DAPI. Negative controls were obtained by omitting the primary antibody on adjacent sections. Samples were examined using an inverted confocal laser microscope (LSM 880; Zeiss).

Cell culture and incubation. Conditionally immortalized human podocytes (provided by P. Mathieson and M.A. Saleem, Children's Renal Unit and Academic Renal Unit, University of Bristol, Southmead Hospital, Bristol, United Kingdom) were differentiated and grown as described (42). Briefly, cells were cultured under growth-permissive conditions at $33^{\circ} \mathrm{C}$ in RPMI 1640 medium (Invitrogen, $11.1 \mathrm{mM}$ glucose) supplemented with $10 \%$ fetal bovine serum (FBS), ITS 100× (insulin, transferrin, and sodium selenite; Invitrogen), and $100 \mathrm{U} / \mathrm{ml}$ penicillin plus $0.1 \mathrm{mg} / \mathrm{ml}$ streptomycin (Sigma-Aldrich). To induce differentiation, podocytes were grown on rat collagen type I (BD Biosciences) and maintained in nonpermissive conditions at $37^{\circ} \mathrm{C}$ for at least 12 days. Two hours before and during the experiments, podocytes were maintained in RPMI 1640 medium with 1\% FBS. Podocytes were incubated with dapagliflozin (10 nM) 2 hours before and during 6 hours of exposure to human serum albumin $(10 \mathrm{mg} / \mathrm{ml}$, Sigma-Aldrich). Human RPTECs (Lonza Group Ltd) were used as positive control for Western blot analysis of SGLT2 expression. The cells were grown in Renal Epithelial Cell Basal Medium (REBM) supplemented with REGM SingleQuots

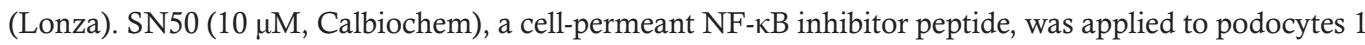
hour before and during 3 hours of exposure to human serum albumin $(10 \mathrm{mg} / \mathrm{ml})$.

Gene silencing of SGLT2. Podocytes were transfected with Silencer Select Pre-designed siRNA for SGLT2 (15 nM) specific for the target sequence NM_003041.3 (siRNA ID s534030, Thermo Fisher Scientific), or with a nontargeting negative control siRNA (Silencer Select Negative Control No. 1 siRNA, Ambion) using Lipofectamine 2000 Reagent (Thermo Fisher Scientific) according to the manufacturer's protocol. Twenty-four hours after transfection, cells were exposed to human serum albumin $(10 \mathrm{mg} / \mathrm{ml})$ for 3 and 6 hours and then collected to evaluate SGLT2 mRNA ( 3 and 6 hours) and protein ( 6 hours) expression.

Western blot analysis. Protein cell lysates were prepared from podocytes or RPTECs using a lysis buffer containing $20 \mathrm{mM}$ Tris- $\mathrm{HCl}$ ( $\mathrm{pH}$ 7.5), $150 \mathrm{mM} \mathrm{NaCl}, 5 \mathrm{mM}$ EDTA, 1\%Triton X-100, 0.1\% SDS, and $1 \%$ sodium deoxycholate supplemented with a protease and phosphatase inhibitor cocktail (Thermo Fisher Scientific). Protein concentration was determined using the BCA Protein Assay (Thermo Fisher Scientific). Equal amounts of protein $(50 \mu \mathrm{g})$ were resolved in 10\% SDS-PAGE under reducing conditions and transferred to nitrocellulose membranes. After blocking, the blots were incubated overnight at $4^{\circ} \mathrm{C}$ with rabbit anti-SGLT2 (1:200, ab85626, Abcam) antibody followed by the appropriate IRDye secondary antibody (1:9,000; 926_32211, LI-COR Biosciences). A rabbit anti-actin antibody (1:3,000; 20-33, Sigma-Aldrich) was used to control loading differences. The membranes were imaged with the Odyssey Fc Infrared image system (LI-COR Biosciences). Relative band intensities were quantified by Image Studio Software (LI-COR Biosciences) and normalized to actin levels.

Quantitative (q) RT-PCR analysis. Total RNA was isolated from human podocytes using TRIzol Reagent (Life Technologies) following the manufacturer's instructions. After treatment with DNase (Promega), cDNA was prepared using SuperScript VILO cDNA synthesis kit (Life Technologies). qRT-PCR analyses were performed on ABI ViiA7 Real-Time PCR System (Life Technologies) using SYBR Green PCR Master Mix (Life Technologies) and the following intron-spanning primers (300 nM): human SGLT2 forward 5'-GGGTTACGCCTTCCACGAG-3' and reverse 5'-AGATGTTTCCCACGGCTGG-3' and human hypoxanthine phosphoribosyltransferase 1 (HRPT1) forward 5'-GGCAGTATAATCCAAAGATGGTCA-3' and reverse 5'-TCCTTTTCACCAGCAAGCTTG-3'. SGLT2 primers were designed on the human solute carrier family 5 member 2 (SLC5A2), transcript variant 1, mRNA reference sequence (NM_003041.3 at the National Center for Biotechnology Information).

Gene expression levels of SGLT2 were normalized to the housekeeping gene HPRT1. Relative quantities were calculated by the $2^{-\Delta \Delta \mathrm{Ct}}$ method. Data are presented as fold change relative to the corresponding control cells. The identity of amplification product was confirmed by sequencing. Starting from podocyte cDNA, a portion of SGLT2, which comprises the qRT-PCR product, was amplified and sequenced in the 2 directions on an ABI 3730 DNA Analyzer (Applied Biosystems). The position and sequence of the primers is shown in Supplemental Figure 3A. Alignment of the exon 6/7 junction with the human SLC5A2 mRNA reference sequence (NM_003041.3) confirmed the specific amplification of the SGLT2 transcript (Supplemental Figure 3B). 
Immunofluorescence analyses. To study SGLT2 expression, human podocytes and human RPTECs, as positive control, were fixed in $2 \%$ paraformaldehyde and $4 \%$ sucrose (Sigma-Aldrich). Nonspecific binding sites were blocked with $2 \%$ FCS, $2 \%$ BSA, and $0.2 \%$ bovine gelatine in PBS. Then, cells were incubated with rabbit anti-SGLT2 antibody (1:200, ab85626, Abcam), followed by a goat anti-rabbit Cy3-conjugated secondary antibody (1:80, 111-165-003, Jackson ImmunoResearch Laboratories). Nuclei were counterstained with the nuclear marker DAPI. Coverslips were examined using a confocal inverted laser microscope.

To evaluate F-actin remodeling and $\alpha$-actinin- 4 and $\beta 1$-integrin expression, podocytes were fixed in $2 \%$ paraformaldehyde plus $4 \%$ sucrose in PBS for 8 minutes at $37^{\circ} \mathrm{C}$ and then permeabilized with $0.3 \%$ Triton X-100 (Sigma-Aldrich) in PBS for 3 minutes at room temperature. Nonspecific binding sites were saturated with blocking solution (2\% FBS, $2 \%$ BSA in PBS) for 1 hour at room temperature. For F-actin staining, podocytes were incubated with $20 \mathrm{U} / \mathrm{ml}$ rhodamine-phalloidin for 45 minutes (Molecular Probes Inc). For $\alpha$-actinin- 4 and $\beta 1$-integrin staining, podocytes were incubated for 1 hour with rabbit anti- $\alpha$-actinin-4 antibody (1:200, EPR2533(2), Origene) or with rat anti- $\beta 1$-integrin antibody (undiluted, AIIB2, Developmental Studies Hybridoma Bank, University of Iowa, Iowa City, Iowa, USA) followed by a goat anti-rabbit Cy3-conjugated secondary antibody (1:80, 111-165-003, Jackson ImmunoResearch Laboratories). Nuclei were counterstained with DAPI. To quantify the number of cells with peripheral F-actin distribution, 15 random images for each sample were acquired by an inverted confocal laser scanning microscope (LSM $510 \mathrm{Meta}$ ) and the percentage of cells with F-actin rearrangement with respect to total cells for each field was then evaluated. The quantification of $\alpha$-actinin- 4 remodeling was performed in 15 random fields per sample and expressed as percentage of podocytes with $\alpha$-actinin-4 filamentous fibers. The $\beta 1$-integrin staining was examined using ApoTome Axio Imager Z2, and the expression was evaluated in 15 random fields per sample by using ImageJ software (NIH) and expressed as fluorescent pixels per cell. Analyses for cytoskeletal remodeling studies were performed by an individual blinded to treatment group.

Patients. Renal tissues of patients with idiopathic membranous nephropathy from the archives of the Unit of Nephrology, Azienda Ospedaliera Papa Giovanni XXIII (Bergamo, Italy) were studied. Demographic and clinical parameters (systolic and diastolic blood pressure, proteinuria and serum creatinine levels) at the time of renal biopsy were retrieved from the hospital database. Specimens of uninvolved portions of kidney collected from tumor nephrectomy $(n=3)$ were used as controls.

Statistics. Results are expressed as means \pm SEM. Data analysis was performed with Prism software (GraphPad Software Inc.). Comparisons were made using ANOVA with the Tukey's multiple-comparisons post hoc test, or Student's $t$ test as appropriate. Statistical significance was defined as $P<0.05$.

Study approval. All procedures involving animals were performed in accordance with institutional guidelines in compliance with national (D.L.n.26, March 4, 2014), and international laws and policies (directive 2010/63/EU on the protection of animals used for scientific purposes) and were approved by the Institutional Animal Care and Use Committees of IRCCS - Istituto di Ricerche Farmacologiche Mario Negri. All experimental protocols involving human subjects and requiring informed consent were carried out in accordance with the Declaration of Helsinki and good clinical practice guidelines, and approved by the Ethical Committee of the Azienda Socio Sanitaria Territoriale (ASST) Papa Giovanni XXIII.

\section{Author contributions}

PC, C. Zoja, AB, and GR conceived and designed the study, discussed data, and wrote the manuscript. ML, D. Cerullo, D. Corna, SB, and C. Zanchi performed experiments, analyzed data, and revised the manuscript. SV performed experiments and analyzed data. MM discussed data and revised the manuscript. All authors approved the final version of the manuscript.

\section{Acknowledgments}

This study was supported by a grant (NCR-16-12066) from AstraZeneca Lab Italia. The authors wish to thank Flavio Gaspari for helpful advice, and Antonio Cannata, Silvia Ferrari, Nadia Stucchi, and Fabiola Carrara for technical assistance. Manuela Passera and Kerstin Mierke helped to prepare and edit the manuscript. Monica Locatelli is a recipient of a fellowship from Fondazione Aiuti per la Ricerca sulle Malattie Rare (A.R.M.R.), Bergamo, Italy. Domenico Cerullo is a recipient of a fellowship from Fondazione Istituti Educativi di Bergamo, Bergamo, Italy. 
Address correspondence to: Carlamaria Zoja, IRCCS - Istituto di Ricerche Farmacologiche Mario Negri, Centro Anna Maria Astori, Science and Technology Park Kilometro Rosso, via Stezzano, 87, 24126 Bergamo, Italy. Phone: 39.035.42131; Email: carlamaria.zoja@marionegri.it.

1. Vallon V, Thomson SC. Targeting renal glucose reabsorption to treat hyperglycaemia: the pleiotropic effects of SGLT2 inhibition. Diabetologia. 2017;60(2):215-225.

2. Perico N, Ruggenenti P, Remuzzi G. ACE and SGLT2 inhibitors: the future for non-diabetic and diabetic proteinuric renal disease. Curr Opin Pharmacol. 2017;33:34-40.

3. DeFronzo RA, Norton L, Abdul-Ghani M. Renal, metabolic and cardiovascular considerations of SGLT2 inhibition. Nat Rev Nephrol. 2017;13(1):11-26.

4. van Bommel EJ, Muskiet MH, Tonneijck L, Kramer MH, Nieuwdorp M, van Raalte DH. SGLT2 inhibition in the diabetic kidney-from mechanisms to clinical outcome. Clin J Am Soc Nephrol. 2017;12(4):700-710.

5. Ruggenenti P, Cravedi P, Remuzzi G. Mechanisms and treatment of CKD. J Am Soc Nephrol. 2012;23(12):1917-1928.

6. Ruggenenti P, et al. Glomerular hyperfiltration and renal disease progression in type 2 diabetes. Diabetes Care. 2012;35(10):2061-2068.

7. Škrtić M, Cherney DZ. Sodium-glucose cotransporter-2 inhibition and the potential for renal protection in diabetic nephropathy. Curr Opin Nephrol Hypertens. 2015;24(1):96-103.

8. Neumiller JJ, Alicic RZ, Tuttle KR. Therapeutic considerations for antihyperglycemic agents in diabetic kidney disease. $J \mathrm{Am}$ Soc Nephrol. 2017;28(8):2263-2274.

9. Toto RD. SGLT-2 inhibition: A potential new treatment for diabetic kidney disease? Nephron. 2017;137(1):64-67.

10. Wanner C, et al. Empagliflozin and progression of kidney disease in type 2 diabetes. N Engl J Med. 2016;375(4):323-334.

11. Neal B, et al. Canagliflozin and cardiovascular and renal events in type 2 diabetes. N Engl J Med. 2017;377(7):644-657.

12. Heerspink HJ, Desai M, Jardine M, Balis D, Meininger G, Perkovic V. Canagliflozin slows progression of renal function decline independently of glycemic effects. J Am Soc Nephrol. 2017;28(1):368-375.

13. Vallon $\mathrm{V}$, et al. SGLT2 inhibitor empagliflozin reduces renal growth and albuminuria in proportion to hyperglycemia and prevents glomerular hyperfiltration in diabetic Akita mice. Am J Physiol Renal Physiol. 2014;306(2):F194-F204.

14. Gembardt F, et al. The SGLT2 inhibitor empagliflozin ameliorates early features of diabetic nephropathy in BTBR ob/ob type 2 diabetic mice with and without hypertension. Am J Physiol Renal Physiol. 2014;307(3):F317-F325.

15. Terami N, et al. Long-term treatment with the sodium glucose cotransporter 2 inhibitor, dapagliflozin, ameliorates glucose homeostasis and diabetic nephropathy in db/db mice. PLOS ONE. 2014;9(6):e100777.

16. Wang XX, et al. SGLT2 protein expression is increased in human diabetic nephropathy: SGLT2 protein inhibition decreases renal lipid accumulation, inflammation, and the development of nephropathy in diabetic mice. J Biol Chem. 2017;292(13):5335-5348.

17. Rajasekeran H, Cherney DZ, Lovshin JA. Do effects of sodium-glucose cotransporter-2 inhibitors in patients with diabetes give insight into potential use in non-diabetic kidney disease? Curr Opin Nephrol Hypertens. 2017;26(5):358-367.

18. Morigi M, et al. A previously unrecognized role of C3a in proteinuric progressive nephropathy. Sci Rep. 2016;6:28445

19. Abbate M, et al. Complement-mediated dysfunction of glomerular filtration barrier accelerates progressive renal injury. $J \mathrm{Am}$ Soc Nephrol. 2008;19(6):1158-1167.

20. Sachs N, Sonnenberg A. Cell-matrix adhesion of podocytes in physiology and disease. Nat Rev Nephrol. 2013;9(4):200-210.

21. Perico L, Conti S, Benigni A, Remuzzi G. Podocyte-actin dynamics in health and disease. Nat Rev Nephrol. 2016;12(11):692-710.

22. Morigi M, et al. In response to protein load podocytes reorganize cytoskeleton and modulate endothelin-1 gene: implication for permselective dysfunction of chronic nephropathies. Am J Pathol. 2005;166(5):1309-1320.

23. Dandapani SV, et al. Alpha-actinin-4 is required for normal podocyte adhesion. J Biol Chem. 2007;282(1):467-477

24. Michaud JL, Chaisson KM, Parks RJ, Kennedy CR. FSGS-associated alpha-actinin-4 (K256E) impairs cytoskeletal dynamics in podocytes. Kidney Int. 2006;70(6):1054-1061.

25. Zanchi C, et al. Fractalkine and CX3CR1 mediate leukocyte capture by endothelium in response to Shiga toxin. J Immunol. 2008;181(2):1460-1469.

26. Delimont D, et al. Laminin a2-mediated focal adhesion kinase activation triggers Alport glomerular pathogenesis. PLoS One. 2014;9(6):e99083.

27. Zhang Y, Thai K, Kepecs DM, Gilbert RE. Sodium-glucose linked cotransporter-2 inhibition does not attenuate disease progression in the rat remnant kidney model of chronic kidney disease. PLoS One. 2016;11(1):e144640.

28. Nakamura N, Masuda S, Takahashi K, Saito H, Okuda M, Inui K. Decreased expression of glucose and peptide transporters in rat remnant kidney. Drug Metab Pharmacokinet. 2004;19(1):41-47.

29. Geerlings S, Fonseca V, Castro-Diaz D, List J, Parikh S. Genital and urinary tract infections in diabetes: impact of pharmacolog ically-induced glucosuria. Diabetes Res Clin Pract. 2014;103(3):373-381.

30. Rizzi M, Trevisan R. Genitourinary infections in diabetic patients in the new era of diabetes therapy with sodium-glucose cotransporter-2 inhibitors. Nutr Metab Cardiovasc Dis. 2016;26(11):963-970.

31. Lewko B, et al. Characterization of glucose uptake by cultured rat podocytes. Kidney Blood Press Res. 2005;28(1):1-7.

32. Coward RJ, et al. The human glomerular podocyte is a novel target for insulin action. Diabetes. 2005;54(11):3095-3102.

33. Piwkowska A, Rogacka D, Angielski S, Jankowski M. Insulin stimulates glucose transport via protein kinase G type I alphadependent pathway in podocytes. Biochem Biophys Res Commun. 2014;446(1):328-334.

34. Tolvanen TA, Dash SN, Polianskyte-Prause Z, Dumont V, Lehtonen S. Lack of CD2AP disrupts Glut4 trafficking and attenuates glucose uptake in podocytes. J Cell Sci. 2015;128(24):4588-4600.

35. Lewko B, Bryl E, Witkowski JM, Latawiec E, Angielski S, Stepinski J. Mechanical stress and glucose concentration modulate glucose transport in cultured rat podocytes. Nephrol Dial Transplant. 2005;20(2):306-311.

36. Reidy K, Kang HM, Hostetter T, Susztak K. Molecular mechanisms of diabetic kidney disease. J Clin Invest. 2014;124(6):2333-2340. 
37. Dai H, Liu Q, Liu B. Research progress on mechanism of podocyte depletion in diabetic nephropathy. J Diabetes Res. 2017;2017:2615286.

38. Lv Z, et al. Fyn mediates high glucose-induced actin cytoskeleton reorganization of podocytes via promoting ROCK activation in vitro. J Diabetes Res. 2016;2016:5671803.

39. Yuen DA, et al. eNOS deficiency predisposes podocytes to injury in diabetes. J Am Soc Nephrol. 2012;23(11):1810-1823.

40. Luis-Lima S, et al. Iohexol plasma clearance, a simple and reliable method to measure renal function in conscious mice. Pflugers Arch. 2016;468(9):1587-1594.

41. Macconi D, et al. Pathophysiologic implications of reduced podocyte number in a rat model of progressive glomerular injury. Am J Pathol. 2006;168(1):42-54.

42. Saleem MA, et al. A conditionally immortalized human podocyte cell line demonstrating nephrin and podocin expression J Am Soc Nephrol. 2002;13(3):630-638. 